\title{
One-step direct reconstitution of biomembranes onto cationic organic polymer bead supports
}

\author{
Samuel Osei-Asante, Mamoru Haratake*, Takeshi Fuchigami, Morio Nakayama**
}

Graduate School of Biomedical Sciences, Nagasaki University, Nagasaki, 852-8521 Japan

Abbreviations: AE1, anion exchanger 1; DAN, 2,3-diaminonaphthalene; DIDS, 4,4'-diisothiocyano2,2'-stilbene disulfonate; DTNB, 5,5'-dithiobis (2-nitrobenzoic acid); GPA, glycophorin A; $\beta$-NADH, $\beta$-Diphosphopyridine nucleotide disodium salt in reduced form; NBD-PE, $N$-4-nitrobenzo-2-oxa-1,3diazole-phosphatidylethanolamine; RBC, red blood cell; SLM, supported lipid membrane; 5P8, $5 \mathrm{mM}$ sodium phosphate solution $(\mathrm{pH} 8)$.

* Corresponding author. Fax: +8195819 2893
** Corresponding author. Fax: +8195819 2893
E-mail addresses: haratake@nagasaki-u.ac.jp (M. Haratake), morio@nagasaki-u.ac.jp (M.

Nakayama)

\begin{abstract}
In this study, we addressed the straightforward reconstitution of red blood cell (RBC) membranes on the surface of cationic organic polymer beads. The RBC membrane-bead complex was obtained by the incubation of white, unsealed rat RBC ghost membranes with a nonporous quaternary ammonium type anion-exchange polymer bead with a $350-550 \mu \mathrm{m}$ diameter. Confocal microscopic observations using a fluorescence membrane probe revealed that the RBC membranes were reconstituted on the outer surface of the bead without any remarkable structural gaps in the membrane. The absences of activity of two peripheral enzymes that latently reside on the cytoplasmic face of the RBC membranes demonstrated that the orientation of the RBC membranes immobilized on the beads was asymmetric as well as that in the native state. The RBC membrane-polymer bead complex was incubated with a primary antibody that is directed against the amino-terminal extracellular domain of the integral protein glycophorin A (GPA). The resulting complex was further incubated with a fluorescent secondary antibody and then subjected to confocal microscopic observations. Fluorescence resulting in the binding of the secondary antibody was found on the surface of
\end{abstract}


the complex, which indicates that the amino-terminal extracellular domain of GPA is exposed to the surface of the complex. In addition, the anion uptake function of the most abundant integral protein anion exchanger 1 (AE1) immobilized on the polymer beads was inhibited by pretreatment with its specific inhibitor 4,4'-diisothiocyano-2,2'-stilbene disulfonate, as is observed for the intact RBCs. Based on all these results, the RBC membranes were thought to be reconstituted on the ionic polymer beads by our one-pot procedure while maintaining the orientation and functions of the membrane proteins to some extent.

Keywords: biomembrane; red blood cell; reconstitution; ionic polymer; integral protein.

\section{Introduction}

Biomembranes surrounding discrete biological compartments in living systems are composed of protein and lipid molecules held together by noncovalent interactions [1]. They do not only act as a physical barrier, but also play diverse physiological roles. Many of them have been elucidated using various in situ biochemical techniques over the past few decades $[2,3]$. Meanwhile, numerous model systems have been developed for the study of the structural and functional features of biomembranes, because mutual dependence between the lipid and protein molecules appears to be highly complex and difficult to directly study in living cells. A notable example of these models is the supported lipid membrane (SLM) on solid materials, such as silica and glass beads [4-8]. The SLM systems have contributed to the elucidation of many membrane processes since their discovery about a quarter of a century ago; for instance, in vitro studies of membrane-cytoskeleton interactions using biomimetic membranes turned out to be helpful to obtain mechanistic insights into the dynamics of these processes $[9,10]$. Because of the mechanical strength provided by the solid supports, the surface of SLMs can offer the possibility to use many surface sensitive techniques, such as a quartz crystal microbalance and attenuated total reflection Fourier transform infrared spectroscopy $[11,12]$. Another advantage of the SLM systems is their ability to retain the fluidity and lateral mobility of the membranes $[6,13]$. This is thought to be due to a thin layer of water (on the order of 1-2 $\mathrm{nm}$ ) between the bilayer membrane and the support material that acts as a lubricant and allows for the rapid diffusion of the lipids in both membrane leaflets.

So far, most of such model systems have been simply prepared from phospholipid molecules. One of the advanced systems for studying the structures and functions of 
biomembranes involves the incorporation of integral and/or peripheral membrane proteins into an artificial phospholipid-based SLM in the non-denatured state, thus maintaining their asymmetric native orientation [14]. Controlling the orientation and density of the proteins in the membranes still seems to be one of the major setbacks of this approach. An alternative way is to directly immobilize the biomembranes on the solid support materials. Several attempts have been made in this regard with some success; Tanaka et al. reconstituted red blood cell membranes and sarcoplasmic reticulum membranes onto biocompatible planar supports $[15,16]$. However, the same seems to hold for the cytoplasmic face of the membranes that was often exposed to an outside buffer solution, that is, the orientation of the immobilized biomembranes is opposite to that found in the native state [17].

Recently, we developed a novel methodology for the preparation of an ionic polymer bead-supported lipid system, in which the electrostatic attractive interactions between the lipids and ionic polymer beads serve as "molecular glue" to immobilize the lipid bilayer membranes on the beads $[18,19]$. We successfully applied the ionic surface character of the system prepared with an artificial anionic lipid as a stationary phase for ion-exchange chromatography, in which the binding of the anionic lipids to the cationic surface of the polymer beads appeared to lend stability to the supported bilayer structure [20]. In this study, our methodology was extended to the reconstitution of red blood cell plasma membranes on the surface of cationic polymer beads with diameter of several hundreds of micrometers. The location and orientation of the resulting RBC membrane in the cationic polymer beads were identified by confocal laser scanning fluorescence microscopy, and the native function of an integral protein in the membrane was further examined.

\section{Experimental}

\subsection{Materials}

Selenious acid $\left(\mathrm{H}_{2} \mathrm{SeO}_{3}\right)$ was obtained from Kanto Chemical Co., Inc. (Tokyo, Japan). 2,3-Diaminonaphthalene (DAN) was from Tokyo Chemical Industry Co., Ltd. (Tokyo, Japan). 5,5'-Dithiobis (2-nitrobenzoic acid) (DTNB), 4,4'-diisothiocyano-2,2'-stilbene disulfonate (DIDS) and cytochrome c (from bovine heart) were purchased from Sigma Co. (St. Louis, MO, U.S.A.). Acetylthiocholine chloride was obtained from Nacalai Tesque, Inc. (Kyoto, Japan). The $\beta$-diphosphopyridine nucleotide disodium salt in the reduced form ( $\beta$ NADH) was from Wako Pure Chemical Industries, Ltd. (Osaka, Japan). DIAION SA11A 
was obtained from Mitsubishi Chemical Co., which is a non-porous quaternary ammonium type anion-exchange polymer bead with a 350-550 $\mu \mathrm{m}$ diameter and its ammonium nitrogen content is $0.85 \mathrm{mmol} / \mathrm{ml}$ polymer in the wet state. Water used in this study was generated using a Milli-Q Biocel system. All other chemicals were of commercial reagent grade and were used as received.

\subsection{Isolation of red blood cell $(R B C)$ ghost membrane and its reconstitution on $S A 11 A$}

Fresh blood from a 3- to 4-week old male Wistar rat was collected in a heparinized VENOJECT II tube (TERUMO, Tokyo, Japan). The sample was centrifuged at 1,400 $g$ for $10 \mathrm{~min}$ at room temperature, and the plasma and buffy coat were removed by aspiration. The precipitated RBCs were washed three times with isotonic phosphate buffer ( $\mathrm{pH}$ 7.4). The packed RBCs $(1 \mathrm{ml})$ were osmotically hemolyzed by the addition of 40 volumes of a $5 \mathrm{mM}$ cold sodium phosphate solution ( $\mathrm{pH} 8$ ) (5P8). The hemolysate was centrifuged at 22,000 g and $4{ }^{\circ} \mathrm{C}$ for $20 \mathrm{~min}$ and then the supernatant was removed by aspiration. To remove the hemoglobin bound to the membranes, the red pellets were washed three times with 5P8 and then white unsealed RBC ghost membranes were obtained.

The RBC ghost membranes obtained from $1 \mathrm{ml}$ of the packed RBCs were suspended in $10 \mathrm{ml}$ of $5 \mathrm{P} 8$ and then sonicated for $1 \mathrm{~min}$ at $80 \mathrm{~W}$ using a probe-type sonicator 250D (Branson, Danbury, CT, U.S.A.). SA11A was soaked in 5P8 overnight before mixing with the RBC ghost membranes. The mixture of the RBC ghost membranes and SA11A was shaken at $25{ }^{\circ} \mathrm{C}$ and $120 \mathrm{~min}^{-1}$ for $30 \mathrm{~min}$. The resulting RBC membrane-SA11A complexes were washed three times with 5P8 and kept in the washing solution at $4{ }^{\circ} \mathrm{C}$ until used.

\subsection{Confocal laser scanning fluorescence microscopic observation of RBC membrane-} SA11A complex

For the membrane location experiment, the RBC ghost membrane was spiked with 0.1 mol\% N-4-nitrobenzo-2-oxa-1,3-diazole-phosphatidylethanolamine (NBD-PE, Molecular Probes, Inc., Eugene, OR, U.S.A.) for the phospholipids just before sonication, and the RBC membrane-SA11A complex was prepared as described above. Confocal laser scanning fluorescence microscopic observations were performed using an LSM 710 equipped with operation software ZEN 2008 (Carl Zeiss, Inc., Tokyo, Japan). An objective with a 10-fold magnification and a 0.30 numerical aperture was used to detect the fluorescence emission 
excited by an $\operatorname{Ar} 458 \mathrm{~nm}$ laser beam $(25 \mathrm{~mW})$. Stacking of the fluorescence images in the Zaxis direction was obtained by capturing optical slices taken at $10 \mu \mathrm{m}$ intervals through the top to the middle of the complex, and consisted of 15 sequentially collected slices. All the fluorescence images were collected using a 510-600 nm filter set in a $1024 \times 1024$ image size at 12 bits and a scan speed of seven.

For the membrane orientation experiment, the goat polyclonal anti-mouse glycophorin A (GPA) antibody, that is directed against the amino-terminal domain of GPA (Product \#: sc19453 P, Santa Cruz Biotechnology, Inc., Santa Cruz, CA) at a 1:500 dilution was applied to the RBC membrane-SA11A complex, followed by the donkey anti-goat antibody-fluorescein conjugate (Product \#: sc-2024, Santa Cruz Biotechnology, Inc.) at a 1:2,000 dilution. Crosssectional fluorescent images from the secondary antibody were observed using an Ar $488 \mathrm{~nm}$ laser beam $(25 \mathrm{~mW})$ by the LSM 710 under the same conditions.

\subsection{Determination of phosphorous and protein contents of RBC membrane-SA11A complex}

After wet digestion of the lipid vesicle suspensions using a 5:1 mixture by volume of nitric acid and perchloric acid, the determination of the phosphorous content was based on vanadium (V) which is capable of forming a yellow complex with the phosphate ion [21]. The colour produced by the complexation was monitored at $440 \mathrm{~nm}$. The phosphorous amount in the beads was calculated from the difference in the phosphorous concentration of the RBC ghost membrane suspension between before and after the preparation of the RBC membrane-SA11A complex. The protein concentration was determined by Lowry's method using bovine serum albumin as the reference. The amount of proteins bound to the beads was calculated from the difference in the protein concentration of the RBC ghost membrane suspension between before and after the preparation of the complex.

\subsection{Enzyme activity measurements of acetylcholinesterase and NADH-cytochrome $c$} oxidoreductase

For the measurement of the acetylcholinesterase activity [22], the RBC membraneSA11A complex was pre-incubated at $37^{\circ} \mathrm{C}$ for $10 \mathrm{~min}$. One hundred microliters each of 10 mM DTNB and $12.5 \mathrm{mM}$ acetylthiocholine chloride was added to the complex suspension. The mixture was allowed to react for $15 \mathrm{~min}$ at $37{ }^{\circ} \mathrm{C}$, and then the colour generated by the cleavage of the disulfide bond of DTNB was spectrophotometrically monitored at $412 \mathrm{~nm}$. 
The relative activity for the RBC membrane-SA11A complex was calculated using the following equation (substrate accessibility to the enzyme); Relative activity $(\%)=100 \times\left(A_{\mathrm{CPX}}\right.$ $\left.-A_{\mathrm{BLK}}\right) /\left(A_{\mathrm{UGS}}-A_{\mathrm{BLK}}\right)$, where $A_{\mathrm{CPX}}, A_{\mathrm{UGS}}$ and $A_{\mathrm{BLK}}$ are the absorbance values for the complex, ghost membrane and membrane-free blank, respectively. The values of $A_{\mathrm{CPX}}$ and $A_{\mathrm{UGS}}$ were normalized to the protein content in each sample. For the measurement of the NADHcytochrome c oxidoreductase activity [22], fifty microliters of $2 \mathrm{mM} \mathrm{NADH}$ and $300 \mu \mathrm{l}$ of $0.84 \mathrm{mg} / \mathrm{ml}$ cytochrome $\mathrm{c}$ were used in place of DTNB and acetylthiocholine chloride. The colour change due to the consumption of cytochrome $\mathrm{c}$ was monitored at $550 \mathrm{~nm}$. All other conditions were the same as those for acetylcholinesterase.

\subsection{Uptake experiments of selenious acid to RBC membrane-SA11A complex}

The RBC membrane-SA11A complexes were incubated with $8 \mu \mathrm{M}$ selenious acid in 5P8 for $10 \mathrm{~min}$ at $25{ }^{\circ} \mathrm{C}$, and aliquots of the supernatant were then withdrawn. For the DIDS inhibition experiment, the RBC membrane-SA11A complex prepared from the RBC ghost membranes pre-treated with $50 \mu \mathrm{M}$ DIDS for $10 \mathrm{~min}$ prior to hemolysis was used. The selenium concentration in the supernatant was fluorometrically determined by the DAN method subsequent to the wet digestion using a 5:1 mixture by volume of nitric acid and perchloric acid [23]. Uptake of the selenious acid by the RBC membrane-SA11A complex from the RBC ghost membrane treated without DIDS was defined as $100 \%$.

\subsection{Statistical Analysis}

All data are presented as the mean and standard error $(\mathrm{SE})(\mathrm{n} \geq 3)$. Statistical analyses were performed using PRISM 4 (GraphPad Software, Inc., La Jolla, CA, U.S.A.). The multiple mean values were compared by a one or two-way analysis of variance using the Bonferroni post-hoc test. Comparisons were considered statistically significant at $P<0.05$.

\section{Results and discussion}

The RBC membrane was used for the preparation of a biomembrane-SA11A complex, because it is easy to isolate and available in large quantities. It is also one of the best-studied biomembranes [24]. We attempted the reconstitution of the RBC ghost membrane on cationic polymer beads by a previously reported slightly modified procedure [18]. During 
this preparation process, the electrostatic attractive interactions between the negatively charged parts of the RBC ghost membranes and the positively charged SA11A beads were thought to be responsible for the adhesion of the RBC membranes to the beads. The SA11A beads with diameters ranging from 350 to $500 \mu \mathrm{m}$ could be expected to be a better platform for the RBC membranes, because its low surface curvature does not render them physically restrained. Particularly, RBC possesses a flexible membrane structure that can easily deform so as to smoothly pass through the peripheral capillary vessels ( $2-3 \mu \mathrm{m}$ in diameter). In the SLM systems using silica and glass beads, the water layer is often not thick enough to accommodate the cytoplasmic domains of the integral proteins, which is thought to lead to their restricted motion or even denaturation [25]. On the other hand, ionic SA11A beads possess a highly hydrated structure due to the ammonium and its counter ions in the polymer chains, which could possibly provide the immobilization of the cytoplasmic domains of the integral proteins with improved lateral mobility.

How do the RBC ghost membranes actually respond to complexation with SA11A? In particular, does the complexation affect the structural reorganization of the membrane? These questions were first examined by analysis of the location of the RBC ghost membrane bound to SA11A. A fluorescence membrane probe NBD-PE $(0.1 \mathrm{~mol} \%$ for phospholipids in the RBC ghost membrane) was spiked into the RBC ghost membranes during the preparation of the complex, and then the resultant was subjected to confocal laser scanning fluorescence microscopic observations. In the cross-sectional images around the middle of the SA11A bead, the fluorescence from NBD-PE in the RBC ghost membranes was clearly found along the outer surface of the SA11A bead (Fig. 1A). When the set of the cross-sectional images was stacked up in the Z-axis direction, a continuous hemispheric fluorescence image of the SA11A bead was obtained (Fig. 1B). In addition, the NBD-PE molecules did not bind to the naked (RBC membrane-free) SA11A at all (data not shown). Based on these microscopic data, the RBC ghost membranes were thought to be reconstituted around the SA11A bead without any remarkable gaps.

To obtain information on the composition of the RBC membranes immobilized on SA11A, the amounts of phospholipid and protein in the RBC membrane-SA11A complex were determined (Table 1). The phospholipids and proteins from the RBC ghost membranes were evidently bound to SA11A. In our preparation methodology, anionic phospholipids (e.g., phosphatidylserine) and anionic moieties of proteins (e.g., glutamic acid and sialic acid) could preferentially bind to the ammonium cations in SA11A due to the electrostatic 
attractive interactions. The weight ratio of phosphorous to protein is a measure of the material composition of the RBC membrane. The value of the ratio for the $\mathrm{RBC}$ membrane immobilized on SA11A was higher than that for the RBC ghost membrane. The RBC ghost membranes involve cytoskeletal and scaffold proteins, such as ankyrin, actin and spectrin, on the cytoplasmic face of the membranes. These proteins are weakly attached to the RBC plasma membranes compared to the membrane-embedded peripheral and integral proteins. Consequently, such cytoskeletal proteins may be disassembled from the RBC ghost membranes during the complexation process.

In the reconstitution of biomembranes to model systems, it is important whether the membranes are immobilized in the inside-out or the right-side-out (native) orientation. To investigate the orientation of the RBC membrane immobilized on SA11A, we first determined the enzyme activity of the peripheral membrane proteins in the RBC membraneSA11A complex. Both acetylcholinesterase and NADH-cytochrome c oxidoreductase latently reside on the cytoplasmic face of the RBC membranes. The activity of these enzymes ("substrate accessibility") is most commonly used as a measure for assessing the quality of the sealed RBC ghost and sealed RBC inside-out vesicles. The activity of these enzymes was apparently observed for the RBC ghost membranes used for the preparation of the RBC membrane-SA11A complex, while it was almost completely abolished for the RBC membrane-SA11A complex (Fig 2), as is observed for the intact RBCs. These results demonstrate that the orientation of the RBC membranes immobilized on SA11A is asymmetric as well as that in the native state.

The absence of the enzyme activity in the complexes (Fig. 2) may be also explained by the inactivation (denaturation) of these peripheral proteins during the reconstitution process. Second, the membrane orientation of the RBC membranes immobilized on the SA11A was assessed using a GPA-specific primary antibody and a secondary antibody conjugated with fluorescein. GPA is the second most abundant integral protein that is a single polypeptide of 131 amino acids with 16 attached oligosaccharide units outside the cell (Fig. 3A). The RBC membrane-SA11A complex was incubated with a primary antibody that is directed against the amino-terminal extracellular domain of GPA. The resulting complex was further incubated with its fluorescent secondary antibody and was subjected to confocal microscopic observations. When the amino-terminal extracellular domain of GPA is faced with SA11A (the inside-out orientation), the secondary antibody cannot bind to the complex via the primary antibody. Fluorescence resulting from the binding of the secondary antibody was 
clearly found on the surface of the complex (Fig. 3B), which indicates that the aminoterminal extracellular domain of GPA in the RBC membranes on the SA11A beads is exposed to the surface of the complex, that is, the right-side-out orientation.

Denaturing and activity loss of membrane proteins is often one of the drawbacks in the immobilization of biomembranes onto solid support materials [25]. To assess the integrity of the membrane proteins on the RBC membrane-SA11A complex, we measured the activity of anion exchanger 1 (AE1). AE1, a 911-amino acid long glycoprotein, is the most abundant integral protein that shares nearly one-third of the total protein weight in the RBC plasma membrane, being present in approximately $10^{6}$ copies per cell. This protein involves two structurally distinct domains, that is, the amino-terminal domain (M1-P403) is cytosolic with a molecular weight of $43 \mathrm{kDa}$, and the membrane embedded domain with the carboxylterminal tail (Q404-V911, molecular weight of $52 \mathrm{kDa}$ ) traverses the plasma membrane multiple times (Fig. 4A) [26, 27]. The membrane domain also contains a single $N$-linked glycosylation site, N642, on its extracellular surface. Monovalent anions, such as the bicarbonate anion $\left(\mathrm{HCO}_{3}^{-}\right)$, are known to be taken up into the $\mathrm{RBC}$ through $\mathrm{AE} 1$ in the bloodstream [28]. Selenious acid is also known to be one of the substrates of AE1 [29]; actually, when the intact RBCs (hematocrit $20 \%$ by volume) were incubated with $8 \mu \mathrm{M}$ selenious acid in isotonic phosphate buffer $(\mathrm{pH} \mathrm{7.4)}$ for $10 \mathrm{~min}, 94.9 \%$ of the added selenious acid was taken up into the RBCs. The function of AE1 on the RBC membraneSA11A complex was assessed using selenious acid as a substrate of AE1. When the complex was incubated with $8 \mu \mathrm{M}$ selenious acid in $5 \mathrm{mM}$ phosphate solution (pH 8) for $10 \mathrm{~min}, 27.8$ $\pm 1.3 \%$ of the selenious acid was taken up into the complex. Such an uptake of selenious acid into the complex seemed to be partly due to the function of AE1 that was immobilized on the surface of the SA11A. When the RBC membrane-free SA11A was treated with selenious acid under the same conditions, $29.8 \pm 1.1 \%$ of selenious acid added was taken up into SA11A. This binding of selenious acid is simply due to the anion-exchange process. Therefore, anionic selenious acid species can bind to the cationic SA11A via not only the AE1 pathway, but also the simple ion-exchange process, if the RBC membranes on SA11A have tiny physical defects that allow selenious acid to pass through. To verify the contribution of the AE1 pathway to the uptake of selenious acid into the RBC membranesSA11A complex, we also conducted an inhibition experiment using DIDS. The anion exchange function of AE1 is known to be specifically inhibited by the pretreatment of DIDS that covalently bind to the lysine residues (K539 and K851) of AE1 [30]. If most of the 
selenious acid is bound to the RBC membrane-SA11A complex via a simple ion-exchange process, the DIDS inhibitory effect is scarcely observed for the complex. Conversely, if the AE1 pathway contributes to the uptake of the selenious acid, the uptake rate of the selenious acid should be reduced by the DIDS treatment of AE1, as is observed for the intact RBC. The pretreatment of the intact RBC with $50 \mu \mathrm{M}$ DIDS for 10 min led to an $80 \%$ reduction in the uptake of the selenious acid (Fig. 4B), while the uptake rate of the selenious acid into the RBC membranes-SA11A complex decreased to nearly $50 \%$, when it was prepared from the RBC ghost membranes pre-treated with DIDS under the same conditions (Fig. 4C). Thus, the uptake of the selenious acid into the RBC membranes-SA11A complex was thought to be largely dependent on the AE1 pathway. In addition, the exposure of the cytoplasmic domain of AE1 was hardly observed in the complex, when examined by the confocal fluorescence microscopic technique using the anti-AE1 antibody against the amino-terminal cytoplasmic domain and a secondary antibody conjugated with fluorescein (data not shown). Consequently, this evidence also supports the facts that the AE1 protein is immobilized in the native orientation on the surface of SA11A with its physiological activity to some extent.

In summary, we prepared the RBC membrane-ionic bead complex by a one-pot reconstitution method based on the electrostatic attractive interactions. Confocal microscopic images revealed that the RBC membranes were located on the outer surface of the cationic SA11A bead without any remarkable structural gaps in the membrane. The results showing the absences of activity of two peripheral enzymes that latently reside on the cytoplasmic face of the RBC membranes and the specific binding of anti-GPA antibody implied that the RBC membranes are immobilized in the right-side-out orientation on SA11A. Furthermore, the anion uptake function of the integral protein AE1 was, in part, observed in the complex. Consequently, our one-pot procedure for the formation of bilayer membrane structures on ionic polymer beads was extendable to the reconstitution of biomembranes in which the orientation and functions of the membrane proteins are maintained to some extent. Hence, this procedure could possibly provide a system for immobilizing the biomembranes with their native membrane orientation, structures and functions. 


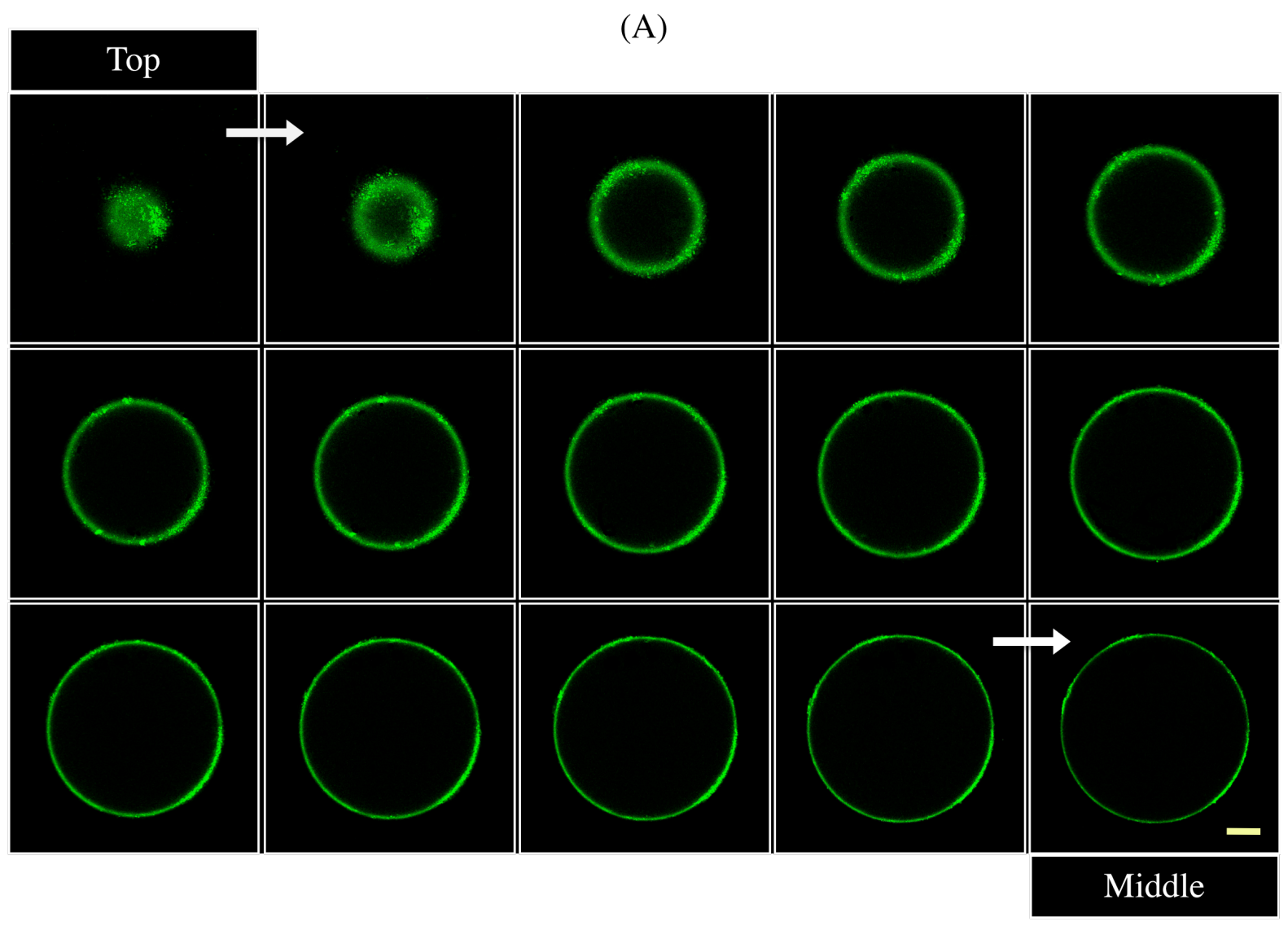

(B)

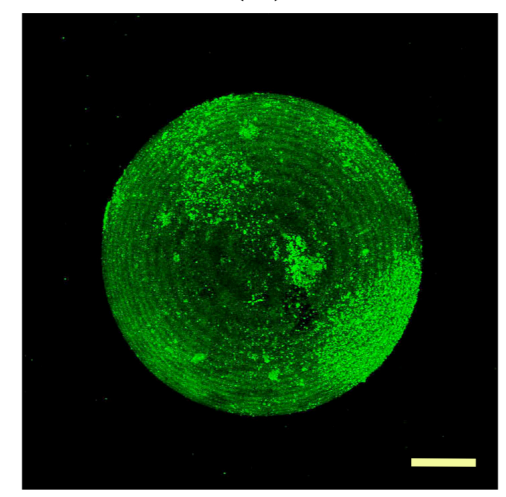

Fig. 1. Confocal fluorescence images of RBC membrane-SA11A complex labeled with NBD-PE. (A) Cross-sectional images, Interval of each section: $10 \mu \mathrm{m}$, (B) Stacked image of (A) in Z-axis direction. NBD-PE concentration: $0.1 \mathrm{~mol} \%$ for phospholipids in the RBC ghost membrane. Pale yellow bar in this panel represents $100 \mu \mathrm{m}$. 


\section{Table 1}

Phosphorous and protein contents in RBC membrane-SA11A complexes and RBC ghost membrane suspension.

\begin{tabular}{|c|c|c|c|}
\hline & $\begin{array}{l}\text { Phosphorous content }{ }^{\text {a }} \\
(\mu \mathrm{g} / \mathrm{ml} \text {-bead or }-\mathrm{RBC})\end{array}$ & $\begin{array}{l}\text { Protein content }^{\mathrm{a}} \\
(\mu \mathrm{g} / \mathrm{ml} \text {-bead or }-\mathrm{RBC})\end{array}$ & $\begin{array}{l}\text { Weight ratio of } \\
\text { phosphorous to } \\
\text { protein }\end{array}$ \\
\hline $\begin{array}{l}\text { RBC membrane- } \\
\text { SA11A complex }\end{array}$ & $49.7 \pm 5.5$ & $159 \pm 13$ & $1: 3.2$ \\
\hline $\begin{array}{l}\text { RBC ghost } \\
\text { membrane }\end{array}$ & $110.5 \pm 17.4$ & $950 \pm 57$ & $1: 8.6$ \\
\hline
\end{tabular}

${ }^{\text {a }}$ Values are mean \pm standard deviation for three independent experiments.

${ }^{b}$ Weight ratio of mean values of the phosphorous and protein contents.

(A)

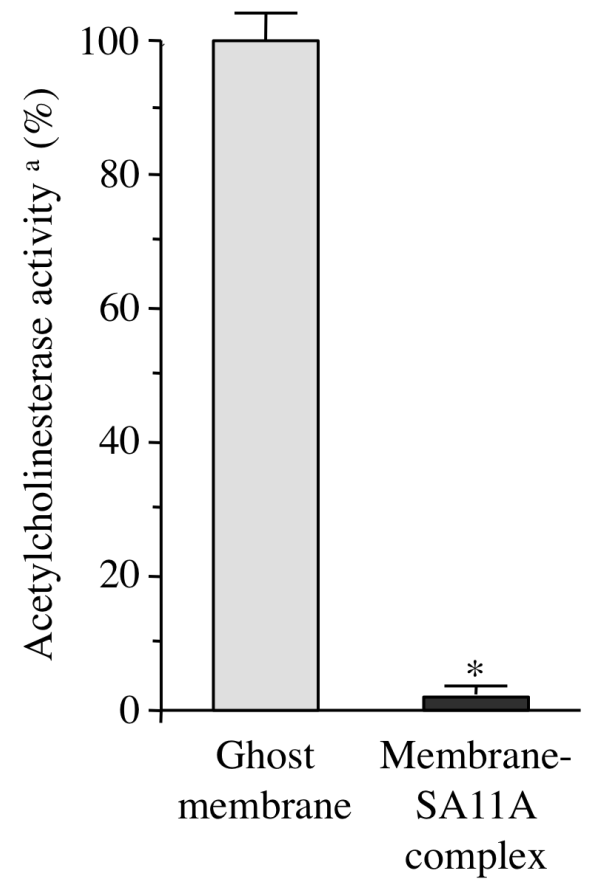

(B)

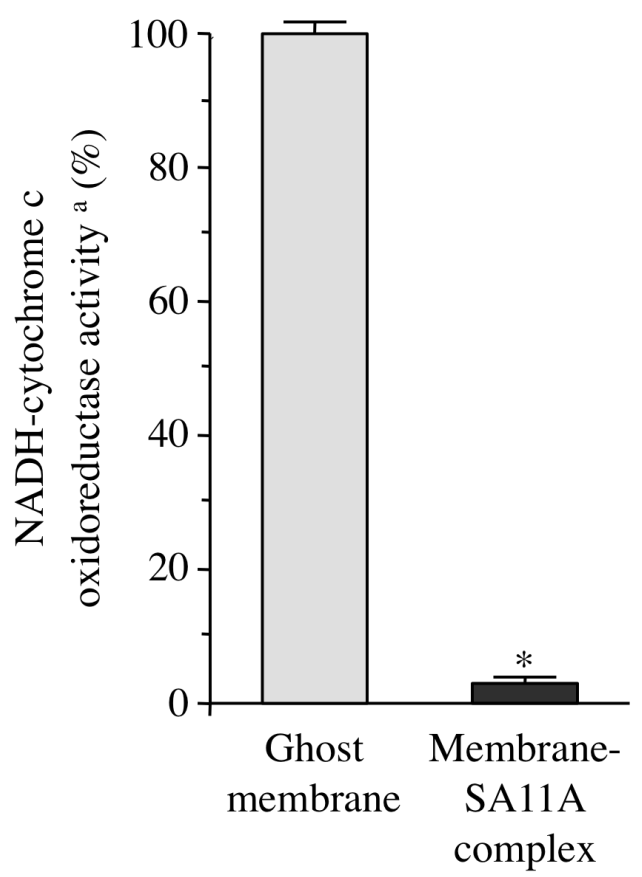

Fig. 2. Relative enzyme activity of acetylcholinesterase (A) and NADH-cytochrome c oxidoreductase (B) in the RBC membrane-SA11A complex. ${ }^{a}$ Enzyme activity of the RBC ghost membranes was defined as $100 \%$. $* P<0.05$. 
(A)

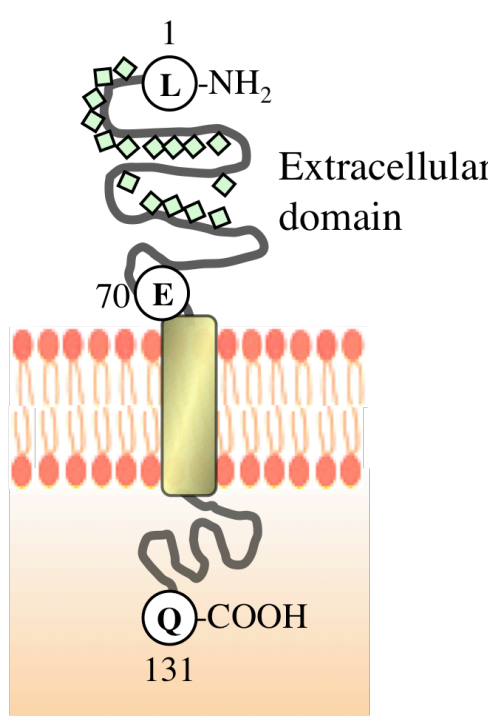

(B)

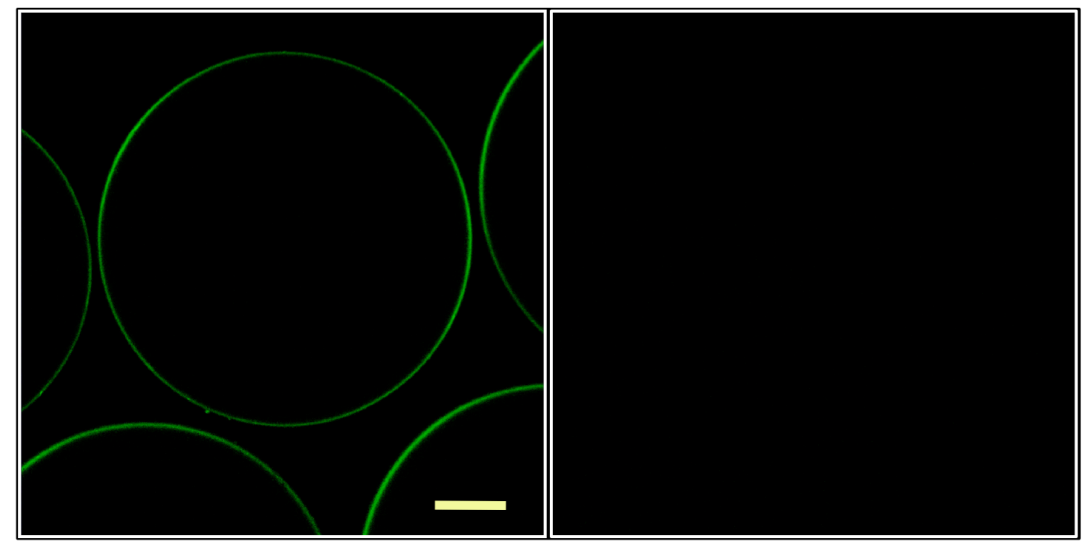

Fig. 3. A topographical model of glycophorin A (A), fluorescence image of RBC membraneSA11A complex after treatment of anti-glycophorin A antibody and fluorescein-labeled secondary antibody (B) and after treatment of fluorescein-labeled secondary antibody only (C). Pale yellow bar in this panel represents $100 \mu \mathrm{m}$. 
(A)

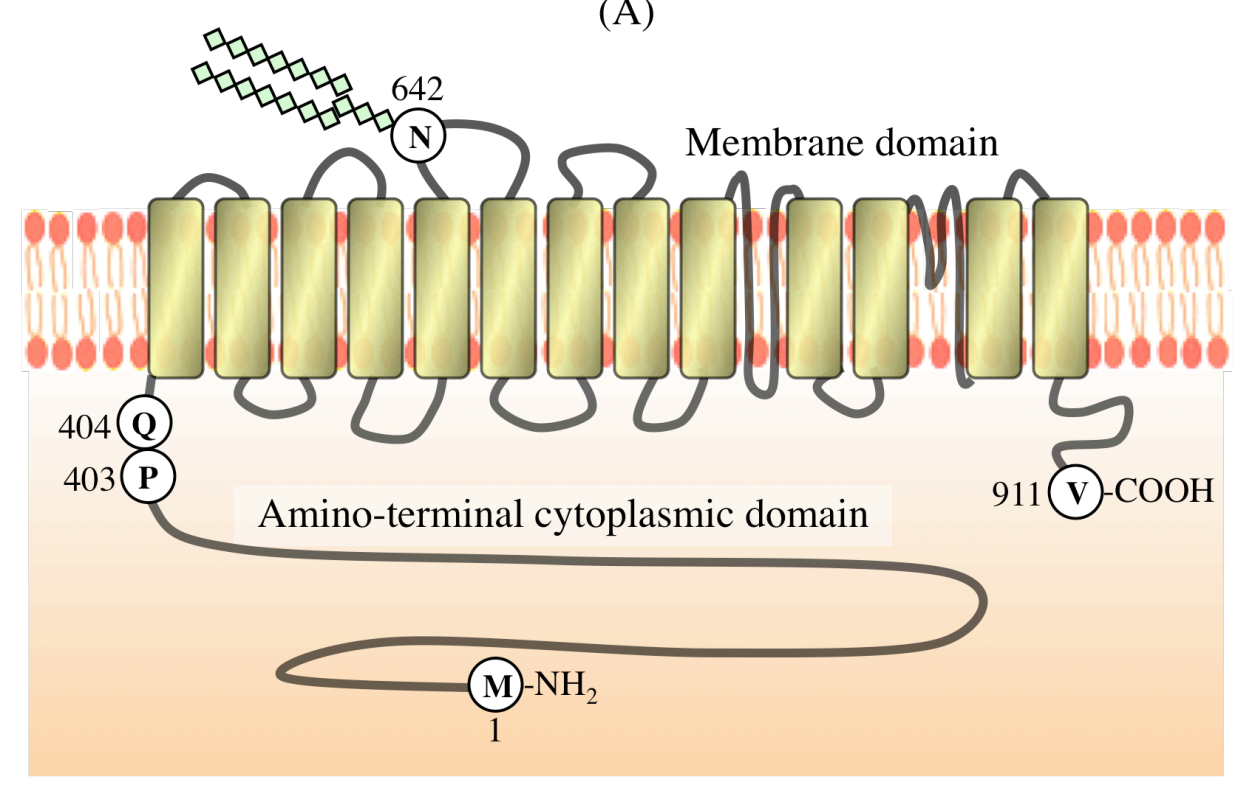

(B)

(C)
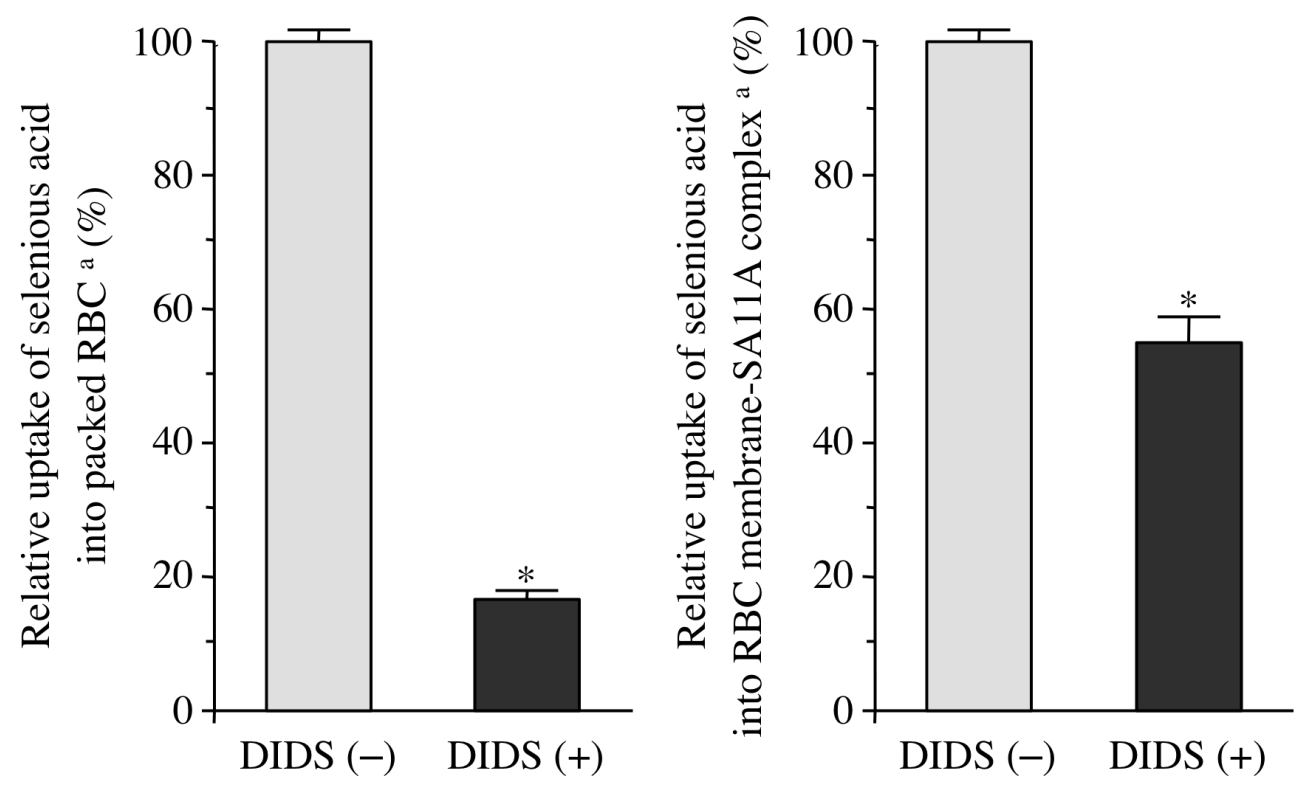

Fig. 4. A topographical model of anion exchanger 1 (A) and uptake of selenious acid by intact RBC (B) and the RBC membrane-SA11A complex (C). Intact RBCs were treated with and without $50 \mu \mathrm{M}$ DIDS in isotonic phosphate buffer ( $\mathrm{pH} 7.4$ ) for $10 \mathrm{~min}$ at $37{ }^{\circ} \mathrm{C}$ prior to hemolysis. ${ }^{a}$ Uptake rates of selenious acid into the respective materials treated without DIDS were defined as $100 \%$. $* P<0.05$. 


\section{References}

[1] S.J. Singer, G.L. Nicolson, Science 175 (1972) 720.

[2] K. Simons, E. Ikonen, Nature 387 (1997) 569.

[3] D.M. Engelman, Nature 438 (2005) 578.

[4] L.K. Tamm, H.M. McConnell, Biophys. J. 47 (1985) 105.

[5] T.M. Bayeri, M. Bloom, Biophys. J. 58 (1990) 357.

[6] R. Lipowsky, Nature 349 (1991) 475.

[7] E. Sackmann, Science 271 (1996) 43.

[8] M.M. Baksh, M. Jaros, J.T. Groves, Nature 427 (2004) 139.

[9] G. J. Doherty and H. T. McMahon, Ann. Rev. Biophys. 37 (2008) 65.

[10] L.K. Tamm, J.T. Groves, J. Struct. Biol. 168 (2009) 1.

[11] C.A. Keller, K. Glasmastar, V.P. Zhdanov, B. Kasemo, Phys. Rev. Lett. 84 (2000) 5443.

[12] S.J. Johnson, T.M. Bayerl, D.C. McDermott,' G.W. Adam, A.R. Rennie, R.K. Thomas, E. Sackmann, Biophys. J. 59 (1991) 289.

[13] G. Gopalakrishnan, I. Rouiller, D.R. Colman, R.B. Lennox, Langmuir 25 (2009) 5455.

[14] F. Giess, M.G. Friedrich, J. Heberle, R.L. Naumann, W. Knoll, Biophys. J. 87 (2004) 3213.

[15] M. Tanaka, S. Kaufmann, J. Nissen, M. Hochrein, Phys. Chem. Chem. Phys. 3 (2001) 4091.

[16] M. Tanaka, A.P. Wong, F. Rehfeldt, M. Tutus, S. Kaufmann, J. Am. Chem. Soc. 126 (2004) 3257.

[17] M. Tanaka, M. Tutus, S. Kaufmann, F.F. Rossetti, E. Schneck, I.M. Weiss, J. Struct. Biol. 168 (2009) 137.

[18] M. Haratake, S. Hidaka, M. Ono, M. Nakayama, J. Colloid Interface Sci. 299 (2006) 924.

[19] S. Osei-Asante, M. Haratake, T. Fuchigami, M. Nakayama, J. Bioact. Compact. Polym. 25 (2010) 455.

[20] M. Haratake, S. Hidaka, M. Ono, M. Nakayama, Anal. Chim. Acta 589 (2007) 76.

[21] R.E. Kitson, M.G. Mellon, Ind. Eng. Chem. Anal. Ed. 16 (1944) 379.

[22] T.L. Steck, J.A. Kant, Methods Enzymol. 31 (1974) 172.

[23] J.H. Watkinson, Anal. Chem. 38 (1966) 92.

[24] N. Mohandas, P.G. Gallagher, Blood 112 (2008) 3939.

[25] M. Loose, P. Schwille, J. Struct. Biol. 168 (2009) 143.

[26] Q. Zhu, D.W.K. Lee, J.R. Casey, J. Biol. Chem. 278 (2003) 3112.

[27] S.H. Chang, P.S. Low, J. Biol. Chem. 278 (2003) 6879.

[28] D.N. Wang, FEBS Lett. 346 (1994) 26.

[29] K.T. Suzuki, Y. Shiobara, M. Itoh, M. Ohmichi, Analyst 123 (1998) 63.

[30] M. Ramjeesingh, A. Gaarn, A. Rothstein, Biochim. Biophys. Acta - Biomembr. 599 (1980) 127. 